\title{
A novel method for the measurement of half-lives and decay branching ratios of exotic nuclei
}

Ivan Miskun $^{1}$, Timo Dickel ${ }^{1,2}$, Israel Mardor ${ }^{3,4, a}$, Christine Hornung ${ }^{1}$, Daler Amanbayev ${ }^{1}$, Samuel Ayet San Andrés ${ }^{1,2}$, Julian Bergmann ${ }^{1}$, Jens Ebert ${ }^{1}$, Hans Geissel ${ }^{1,2}$, Magdalena Górska ${ }^{2}$, Florian Greiner ${ }^{1}$, Emma Haettner ${ }^{2}$, Wolfgang R. Plaß ${ }^{1,2}$, Sivaji Purushothaman ${ }^{2}$, Christoph Scheidenberger ${ }^{1,2}$, Ann-Kathrin Rink ${ }^{1}$, Helmut Weick ${ }^{2}$, Soumya Bagchi ${ }^{1,2,6}$, Paul Constantin ${ }^{5}$, Satbir Kaur ${ }^{6}$, Wayne Lippert ${ }^{1}$, Bo Mei ${ }^{5}$, Iain Moore ${ }^{7}$, Jan-Hendrick Otto ${ }^{1}$, Stephane Pietri ${ }^{2}$, Ilkka Pohjalainen ${ }^{7}$, Andrej Prochazka ${ }^{2}$, Christophe Rappold ${ }^{1,2}$, Moritz P. Reiter ${ }^{1,8}$, Yoshiki K. Tanaka ${ }^{2}$, and John S. Winfield ${ }^{2}$

For the Super-FRS Experiment Collaboration

1 II. Physikalisches Institut, Justus-Liebig-Universität Gießen, 35392 Gießen, Germany

2 GSI Helmholtzzentrum für Schwerionenforschung GmbH, 64291 Darmstadt, Germany

3 Tel Aviv University, 6997801 Tel Aviv, Israel

4 Soreq Nuclear Research Center, 81800 Yavne, Israel

5 IFIN-HH/ELI-NP, 077126, Măgurele - Bucharest, Romania

6 Saint Mary's University, NS B3H 3C3 Halifax, Canada

7 University of Jyväskylä, 40014 Jyväskylä, Finland

8 TRIUMF, BC V6T 2A3 Vancouver, Canada

Received: 22 February 2019 / Revised: 19 June 2019

Published online: 4 September 2019

(c) The Author(s) 2019. This article is published with open access at Springerlink.com

Communicated by R.V.F. Janssens

\begin{abstract}
A novel method for simultaneous measurement of masses, Q-values, isomer excitation energies, half-lives and decay branching ratios of exotic nuclei has been demonstrated. The method includes first use of a stopping cell as an ion trap, combining storage of mother and daughter nuclides for variable durations in a cryogenic stopping cell (CSC), and afterwards the identification and counting of them by a multiplereflection time-of-flight mass spectrometer (MR-TOF-MS). We utilized our method to record the decay and growth of the ${ }^{216} \mathrm{Po}$ and ${ }^{212} \mathrm{~Pb}$ isotopes (alpha decay) and of the ${ }^{119 m 2} \mathrm{Sb}$ isomer $\left(t_{1 / 2}=850 \pm 90 \mathrm{~ms}\right)$ and ${ }^{119 g} \mathrm{Sb}$ isotope (isomer transition), obtaining half-lives consistent with literature values. The amount of nonnuclear-decay losses in the CSC up to $\sim 10 \mathrm{~s}$ is negligible, which exhibits its extraordinary cleanliness. For ${ }^{119} \mathrm{Sb}$ isotopes, we present the first direct measurements of the mass of its ground state, and the excitation energy and decay branching ratios of its second isomeric state $\left({ }^{119 m 2} \mathrm{Sb}\right)$. This resolves discrepancies in previous excitation energy data, and is the first direct evidence that the ${ }^{119 m 2} \mathrm{Sb}$ isomer decays dominantly via $\gamma$ emission. These results pave the way for the measurement of branching ratios of exotic nuclei.
\end{abstract}

\section{Introduction}

Half-lives and branching ratios to various decay channels are important properties of unstable exotic isotopes. They provide vital input to the verification and extension of nuclear structure models throughout the nuclear chart, and to astrophysical nucleosynthesis models [1]. Exotic nuclei, especially far from the stability valley, likely have numerous decay channels, which may include $\alpha$ - and $\beta$-decays, spontaneous fission, direct single- or multi-nucleon emission, and $\beta$-delayed fission and nucleon emission. For a

\footnotetext{
a e-mail: mardor@tauex.tau.ac.il (corresponding author)
}

review on $\beta$-delayed decays and their importance to nuclear physics and astrophysics, see ref. [2].

Half-lives and decay branching ratios are mainly measured by the detection of light particles involved in the decay ( $\alpha, \beta$, nucleons) as a function of time, and the identification of the daughter nuclides via their $\gamma$ lines, as they are usually created in excited states [3-5]. Penning traps are powerful for purification of mother nuclide beams, whose decay can be studied via several post-trap spectroscopy techniques with minimal background [6].

Other methods use in-trap nuclear decay, and include direct mass measurements of mother and/or daugh- 
ter nuclides via a Penning trap [7] or a multi-reflection time-of-flight mass-spectrometer (MR-TOF-MS) [8]. In the storage ring ESR at GSI, beta decays and branching ratios have been measured with cooled beams [9]. It has further been proposed to measure decay branching ratios by a series of gas-filled stopping cells and MR-TOFMSs [10]. In another approach, different decay branches are identified and counted by measuring the momentum of the daughter nuclide via time-of-flight, after decay inside a Paul trap [11].

In this paper we describe a novel method for measuring half-lives and decay branching ratios of exotic nuclei. We demonstrate for the first time use of a stopping cell as an ion trap. Storage times up to $\sim 10 \mathrm{~s}$ are shown, indicating the high cleanliness level of our cell. The method is universal, and can be used for nuclides that decay via one or more of any of the channels that are listed above.

We require only very general prior knowledge of nuclear properties, since all are measured during the experiment. This is accentuated in the current article, where while demonstrating our method, we measured directly for the first time the mass of the ground state, and the excitation energy and decay branching ratios of the second isomeric state $\left({ }^{119 m 2} \mathrm{Sb}, t_{1 / 2}=850 \pm 90 \mathrm{~ms}\right)$ of the ${ }^{119} \mathrm{Sb}$ isotope. This is the first direct evidence that the ${ }^{119 m 2} \mathrm{Sb}$ isomer decays dominantly by $\gamma$ emission and further, our results offer a solution to a long-existing conflict regarding its excitation energy and spin assignment [12-15].

The paper is organised as follows: in sect. 2 we describe our novel method for measuring half-lives and branching ratios in the FRS-IC; we present the results of our work in sect. 3 ; sect. 4 discusses ongoing developments of the method, and lists future possible measurements with it. We conclude in sect. 5 .

\section{Outline of methodology}

Our method is implemented in the Fragment Separator (FRS) Ion Catcher (IC) [16] at the FRS [17], utilizing its gas-filled cryogenic stopping cell (CSC) [18] and MRTOF-MS [19].

The general concept is outlined in fig. 1. A primary beam is accelerated to relativistic energy by the GSI SIS18 synchrotron. Fragments are produced in the target and are separated in-flight via the FRS, generating an ensemble that includes mainly the mother nuclide of interest. This ensemble is stored in the CSC for a controllable duration, while the mother nuclides of interest decay. Due to the high CSC gas density, almost all daughter nuclides are stored when the decay takes place in the CSC bulk. The mother and daughter nuclides are consequently extracted to the MR-TOF-MS, where they are identified by their mass-to-charge ratio and counted. The mother nuclide decay branching-ratios are determined from the ratios between the number of daughter nuclides.

In addition to branching ratios, we simultaneously measure mother and daughter nuclide masses (and thus the mother nuclide Q-values), and the mother nuclide half- life. To reduce systematic errors, half-lives and branching ratios are studied as a function of CSC storage time.

The method described in this work is uniquely-possible with the FRS Ion Catcher, because of the flexible time structure of the beam that is available from the SIS-18 synchrotron, and the high energy of the FRS fragments (resulting from the SIS-18 high primary beam energy, up to $1 \mathrm{GeV} / \mathrm{u}$ for ${ }^{238} \mathrm{U}$ ions). The high energy of the fragments is necessary for obtaining the required fragment separation, which enables the stopping of a clean sample of the ions of interest in the CSC. The beam temporal structure is required in order to have well defined start time of the decay to the daughter nuclides. All stored ions are extracted to the MR-TOF-MS before the next injection of ions in the CSC.

At other in-flight RIB facilities, the primary beam is generated by a cyclotron that provides only a continuous beam at lower energies, e.g. RIKEN with $345 \mathrm{MeV} / \mathrm{u}$. At such energies isotope identification is possible, but without spatial separation at the required level, because isotones cannot be separated [20]. Therefore, implementing there the concept described in this work requires additional instrumentation to pulse and clean the fragment beam. A series of gas-filled stopping cells and MR-TOF-MS has been suggested to facilitate this [10].

\subsection{Obtaining clean mother nuclide samples in the CSC}

A major requirement of our method is to have a relatively clean sample of mother nuclides stopped in the CSC. Our main concern are nuclides that decay into the mother nuclide of interest or its daughter nuclides, because their amount will grow as storage time increases. Neighboring nuclides that are the eventual daughter nuclides of the decay of interest are also a concern, but of a lesser extent. This is because their amount does not increase with storage time, and can thus be measured in advance at a very short storage time, and subtracted from the actual measurements. The nuclides that generate a problematic background for the case of $\beta$-delayed neutron emission are shown in fig. 2. Similar identification of unwanted contaminant nuclides can be deduced for any other nuclear decay process.

The FRS can provide an isotope beam with the required cleanliness due to the high energy of its fragments. This can be achieved by either an achromatic mode and using the slits to block part of the beam [17], or a monochromatic mode that gives a spatial separation in range, taking advantage of the stopping thickness in the CSC (a few $\mathrm{mg} / \mathrm{cm}^{2}$ ), which will stop mainly the nuclide of interest. LISE ++ [21] and MOCADI [22] simulations were performed for several cases. An example of their output for optimized stopping of a ${ }^{137} \mathrm{Sb}$ sample in the CSC is shown in fig. 3. The simulation is for all the nuclides that appear in fig. 2, when the mother nuclide of interest is the ${ }^{137} \mathrm{Sb}$ isotope. The amount of stopped nuclides is given by the integration of each nuclide's range distribution over the CSC range, which is marked with a $10 \mathrm{mg} / \mathrm{cm}^{2}$ shade in 
FRS

In-flight isotope

production and separation,

energy bunching, slowing down
CSC

Mother of interest stopped, contained and decays
MR-TOF-MS

Mother and daughters identification and counting

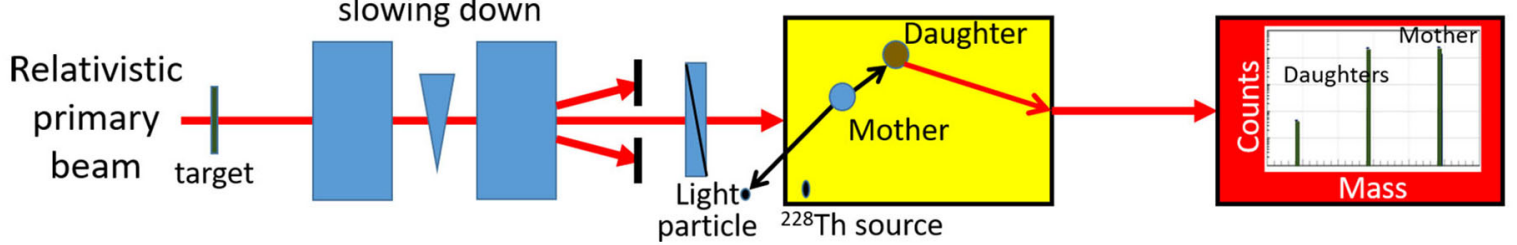

Fig. 1. Scheme of the measurement method. A single decay event inside the CSC (shown in yellow is the active volume of the CSC) and the accumulated mass spectrum in the MR-TOF-MS after the extraction of mother and daughter nuclides are depicted. The internal ${ }^{228} \mathrm{Th} \alpha$-recoil source inside the CSC, used for the off-line measurements in this work, is also shown.

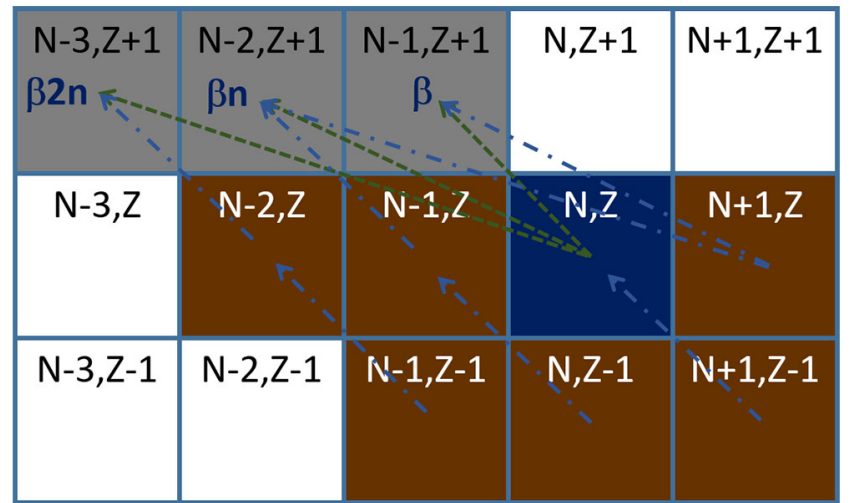

Fig. 2. Visualization of nuclides that generate background for the branching ratio measurement in the case of $\beta$-delayed neutron emission. The dark blue nuclide is the mother nuclide of interest. The gray ones are the daughter nuclides of interest from the possible branches $(\beta, \beta \mathrm{n}, \beta 2 \mathrm{n})$, indicated by dashed arrows. Beam contamination of these daughter nuclides will interfere with the measurement, but can be measured in advance at short storage times and subtracted. Contamination of the brown nuclides, which might decay by $\beta, \beta \mathrm{n}$ and $\beta 2 \mathrm{n}$ to the daughter nuclides of interest (either directly or in a chain) will mask those that result from the mother nuclide of interest, and are hard to account for since their amount grows as a function of storage time. Contamination by white nuclides will not affect the results and can thus be tolerated.

fig. 3. The results are that for $\beta$-delayed neutron emission of ${ }^{137} \mathrm{Sb}$ isotopes, the highest interfering contamination will be the ${ }^{137} \mathrm{Te}$ isotope ( $\beta$-decay daughter) at a level of $10^{-2}$, which can be measured in advance with short CSC storage and subtracted from the actual measurement. Of the other nuclides marked gray or brown in fig. $2,{ }^{136} \mathrm{Sn}$ and ${ }^{137} \mathrm{Sn}$ isotopes are at a level of $10^{-5}$, and all the rest are practically zero. ${ }^{138} \mathrm{Te}$ isotopes are stopped with an amount similar to ${ }^{137} \mathrm{Sb}$ isotopes, but it is one of the nuclides marked white in fig. 2, so its presence does not affect the measurement. Based on these simulations, we set $10^{-4}$ as a conservative lower limit on measurable branching ratios for such decays of neutron-rich isotopes.

Another potential source for contaminants is generated by neutron removal reactions of the incoming beam

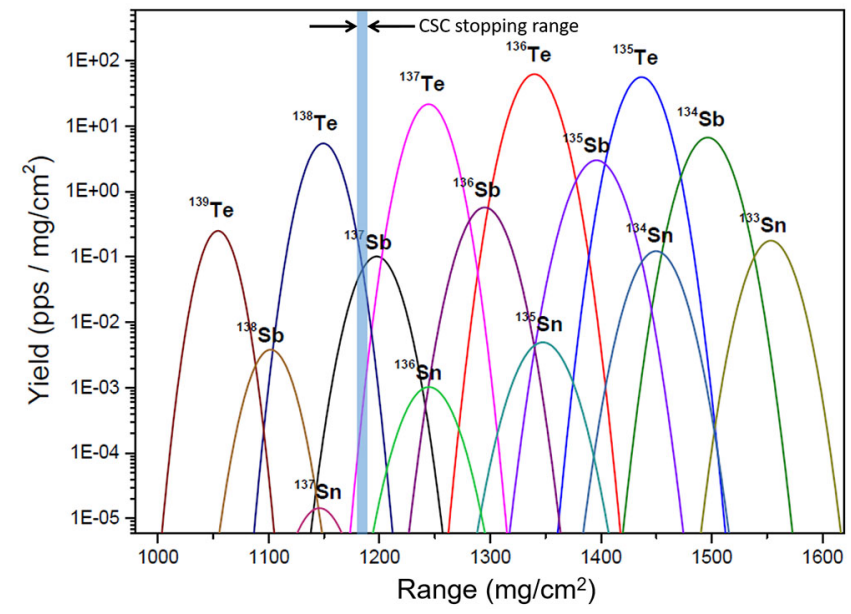

Fig. 3. A LISE ++ simulation of the yields of ${ }^{137} \mathrm{Sb}$ isotopes and all the relevant neighbors according to the scheme of fig. 2, per range in helium. Simulation main settings: $2 \cdot 10^{9}{ }^{238} \mathrm{U}$ ions per second at a primary beam energy of $800 \mathrm{MeV} / \mathrm{u}, \mathrm{Pb}$ target of $2,600 \mathrm{mg} / \mathrm{cm}^{2}$, FRS in monochromatic mode. The shaded area indicates the stopping range distribution in the CSC, $1180-1190 \mathrm{mg} / \mathrm{cm}^{2}$. In practice, the first $1180 \mathrm{mg} / \mathrm{cm}^{2}$ of helium needed to slow down the fragments are emulated by equivalent range of aluminum degraders.

upstream of the CSC. Such reactions generate neutrondeficient isotopes of the investigated mother nuclide. However, the majority of these contamination isotopes will not be stopped in the CSC. The upper limit of this contribution is estimated to be $10^{-4}$, for neutron-rich nuclides.

The lower limit for the measurement of branching ratios will vary for different mother nuclides and decay channels, depending on the positions of the mother and daughter nuclides in the nuclear chart.

\subsection{Daughter nuclides in the CSC}

The range distributions of daughter nuclides in relevant decay processes in $\mathrm{He}$ buffer gas were studied with the stopping and range of ions in matter Monte Carlo program, SRIM [23], using a typical density of 
$40 \mu \mathrm{g} / \mathrm{cm}^{3}$ (namely $4.2 \mathrm{mg} / \mathrm{cm}^{2}$ for the $105 \mathrm{~cm}$ long CSC, which was the areal density for the measurements with online-generated isotopes in this work). For $\gamma$-decay, the daughter nuclide kinetic energy is a few tens of eV, corresponding to range distributions of a few $10^{-2} \mathrm{~mm}$. In $\beta$-decay, the daughter nuclide kinetic energy is a few hundred $\mathrm{eV}$, resulting in a range distribution of $0.05-0.20 \mathrm{~mm}$. When there is also emission of $\beta$-delayed nucleons (or for direct nucleon emission), the daughter nuclide kinetic energy rises to tens of $\mathrm{keV}$, increasing the range distributions to $0.5-2.0 \mathrm{~mm}$. In $\alpha$-decay, the daughter nuclide kinetic energy is in the $100-200 \mathrm{keV}$ range, leading to range distributions around $10 \mathrm{~mm}$ for typical $\alpha$ daughter nuclides.

The CSC inner dimensions are $105 \mathrm{~cm}$ in length and $25 \mathrm{~cm}$ in diameter, so as long as the isotopes of interest are stored and decay in the bulk, there is no risk to lose daughter nuclides on the inner walls. However, in the experiments that are described in this paper, storage of the isotopes took place on the CSC RF carpet close to the exit nozzle (see fig. 1). As described in detail in [18], the pitch of the RF carpet rings is $0.25 \mathrm{~mm}$, and the isotopes are stored in the buffer gas at about half that distance above the RF carpet.

For $\gamma$-decay there is no effect, since the daughter nuclide range distribution is much below $\sim 0.1 \mathrm{~mm}$. On the other hand, for $\alpha$-decay all daughter nuclides in the hemisphere towards the RF carpet will be lost, so one merely needs to double the number of daughter nuclides counted in the MR-TOF-MS to accommodate for this. These are the decay types that were investigated within the current work.

However, for $\beta$-decay and $\beta$-delayed and direct nucleon emission, the daughter nuclide range distribution is in the same order of magnitude as the distance from the RF carpet, rendering a systematic effect that is very difficult to estimate. In sect. 4.1 we describe a solution to this problem, by storage of the isotopes of interest in the bulk for the vast majority of the storage time.

\subsection{Mass measurements and isotope counting}

Mass measurements and isotope counting (absolute intensity) at the FRS-IC are performed with an MR-TOF-MS, which entails a unique combination of performance parameters - fast ( $\sim \mathrm{ms})$, high-resolution (resolving power as high as 600,000 (FWHM)) and non-scanning [19].

The non-scanning nature of the MR-TOF-MS is imperative to our method. It enables the simultaneous measurement of the mother nuclide and all daughter nuclides, which may be removed from each other by up to several mass units. With respect to scanning techniques such as a Penning trap, this saves significant beam-time, and avoids systematic errors that might arise due to sequential measurement of the mother and all daughter nuclides.

The development and validation of the MR-TOF-MS data-analysis procedure is presented in a separate publication [24]. Mass values and amounts of the measured isotopes and their uncertainties are obtained after fitting the mass peaks to a Hyper-EMG function [25]. The procedure allows accurate mass determination even for the most challenging conditions, including very low numbers of events and overlapping mass distributions. In the mass measurements reported in ref. [24], a total relative precision (i.e., centroid uncertainty) down to $6 \cdot 10^{-8}$ was achieved.

\subsection{Half-life and branching ratio measurement}

Half-lives of exotic isotopes are measured directly in the FRS-IC by varying the isotopes' storage time in the CSC. The storage time is varied from its minimal extraction time, tens of milliseconds [16] up to $\sim 10 \mathrm{~s}$ (see sect. 3.2), setting the half-life range that can be measured with this method. This is suitable for a very wide range of exotic isotopes and isomeric states.

Decay branching ratios are derived by calculating the ratios between the amount of the daughter nuclides resulting from a specific decay channel and the total amount of daughter nuclides from all decay channels.

For the measurements with beam-generated isotopes described herein, the spill structure of the heavy-ion synchrotron SIS-18 is adjusted to allow for short (from a few to hundreds of milliseconds, depending on the half-life of the mother nuclide of interest) bunches, separated by long $(\sim 10 \mathrm{~s})$ breaks. This separation is chosen to match the longest anticipated storing time inside the CSC. As a result, short spills of ions are injected into the CSC.

Ion storage in the CSC is achieved by increasing the potential on the extraction nozzle to block extraction for an adjustable duration, and lowering it for a few milliseconds to enable extraction. The storage time is defined as the time between the temporal mid-point of the beam-spill and the start of the low-potential pulse at the extraction nozzle.

For isotopes generated by internal radioactive recoil sources, "spills" are created by pulsing the potential of the source. It is normally negative with respect to the surrounding electrodes, thereby preventing ions from leaving the vicinity $(\sim 1 \mathrm{~cm})$ of the source, and is pulsed periodically to positive values for a few milliseconds.

After extraction from the CSC, the isotopes are identified and counted by analyzing the MR-TOF-MS spectra via the procedure from [24]. The mother nuclide's halflife is extracted by fitting the mother $(P(t))$ and daughter $\left(R_{i}(t)\right)$ counts to the well-known solutions of the Bateman equations $[26]$ :

$$
\begin{aligned}
P(t) & =A_{p} \cdot e^{-\lambda_{P} \cdot t} \\
R_{i}(t) & =y_{0 i} \cdot e^{-\lambda_{R i} \cdot t}+\frac{\lambda_{P}}{\lambda_{R i}-\lambda_{P}} \cdot n_{r i} \cdot A_{p} \cdot\left(e^{-\lambda_{P} \cdot t}-e^{-\lambda_{R i} \cdot t}\right)
\end{aligned}
$$

where $A_{p}$ is the initial amount of the mother nuclide, $\lambda_{P}$ is the mother nuclide's decay constant, related to its halflife by $\lambda_{P}=\frac{\ln (2)}{t_{1 / 2}^{P}}, \lambda_{R i}=\frac{\ln (2)}{t_{1 / 2}^{R i}}$ is the $i$-th daughter nuclide 
decay constant, $y_{0 i}$ is a constant contamination of the $i$ th daughter nuclide, and $n_{r i}$ is the $i$-th decay branching ratio. $n_{r i} \cdot A_{p}$ is the amount of the $i$-th daughter nuclide at infinite time (practically, a time much longer than the mother nuclide half-life). The sum of $n_{r i}$ is equal to unity.

In the current work the half-lives of the daughter nuclides are much longer than the mother nuclide's halflife and the storage times, so for our analysis we assume $\lambda_{R} \rightarrow 0$ and use the simplified expression

$$
R_{i}(t)=y_{0 i}+n_{r i} \cdot A_{p} \cdot\left(1-e^{-\lambda_{P} \cdot t}\right) .
$$

Note that in the investigation of $\alpha, \beta$ and direct and $\beta$-delayed nucleon emission, the FRS will provide an essentially contaminant-free ion sample (sect. 2.1). However, in the case of $\gamma$-decay of isomeric states, there will always be a non-zero constant contamination, since there is no way to separate spatially isomeric states from ground states in the FRS (sect. 2.1). The amount of constant contamination $\left(y_{0 i}\right)$ can be obtained directly by a measurement of all daughter nuclide candidates with a storage time much shorter than the mother nuclide half-life.

The mother nuclide half-life and decay branching ratios are obtained via a global fit of the mother nuclide and all daughter nuclide candidates, thereby providing an improved uncertainty, both statistically and systematically. In particular, the statistics in such a measurement remain constant as a function of storage time, whereas when measuring only the mother nuclide, the statistics decrease as less and less mother nuclides are detected.

By "global" we refer to a unified fit of the data sets of both the mother nuclide and all daughter nuclide candidates, using eq. (1) for the mother nuclide data and eq. (2) or eq. (3) for the daughter nuclide candidates data. We apply the following constraints to the global fit: the same decay constant parameter $\left(\lambda_{P}\right)$ for the mother nuclide decay and daughter nuclides growth, all branching ratios $\left(n_{r i}\right)$, constant contaminations $\left(y_{0 i}\right)$ and the mother nuclide initial amount $\left(A_{p}\right)$ are non-negative, and the sum of $A_{p}$ and all $y_{0 i}$ is unity, as well as the sum of all $n_{r i}$.

The mother nuclide's half-life and decay branching ratios can be also extracted from a measurement at only one CSC storage time, provided that the daughter nuclide constant contaminations are negligible or are known, and the daughter nuclide half-lives are relatively long. The mother nuclide half-life is obtained by division of eq. (1) by eq. (3) (setting $y_{0 i}=0$ ) and subsequent algebraic manipulation

$$
t_{1 / 2}=\ln 2 \cdot t_{s} \cdot\left[\ln \frac{1+D}{D}\right]^{-1},
$$

where $D \equiv \frac{P\left(t_{s}\right)}{\Sigma R_{i}\left(t_{s}\right)}$, and $t_{s}$ is the selected CSC storage time. The branching ratio of the $i$-th daughter nuclide is obtained by

$$
n_{r i}=\frac{R_{i}\left(t_{s}\right)}{\Sigma R_{i}\left(t_{s}\right)} .
$$

The statistical uncertainty in the measured half-lives and branching ratios depends on the statistical uncertainty of the isotope counts for each storage time, which are evaluated by the analysis procedure [24].

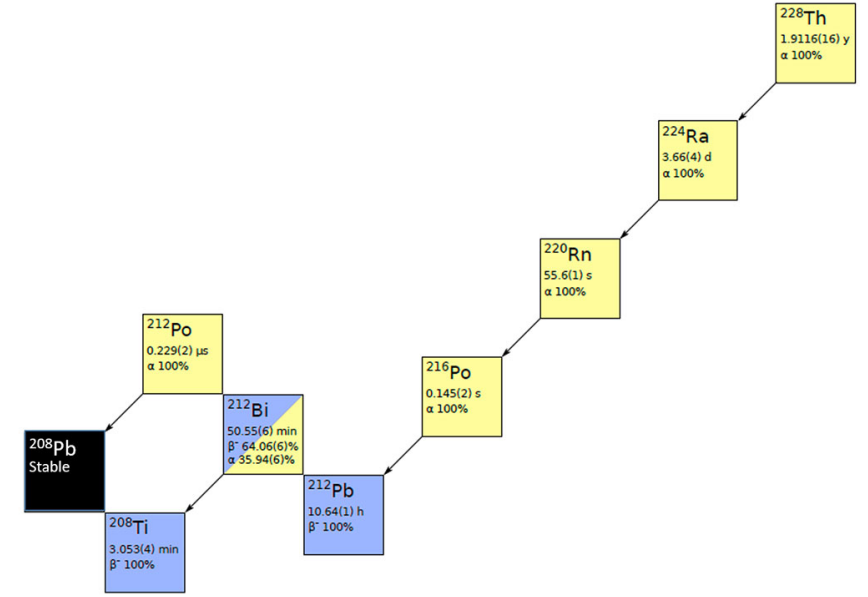

Fig. 4. Decay scheme of the ${ }^{228}$ Th isotope. Yellow: $\alpha$ emitters; blue: $\beta$ emitters; and black: stable isotopes.

A possible source for systematic uncertainties in branching ratio measurements might be loss of particles due to chemical reactions (e.g., charge exchange, molecular formation) in the CSC buffer gas. The results in this paper (sect. 3) indicate that this is a minute effect. In addition, it has been established in previous FRS-IC experiments that the CSC survival and extraction efficiency is essentially element independent, including comparison of a noble element $(\mathrm{Rn})$, and one of the most reactive ones (Th) [27].

Different elements may be extracted with charge states $+1,+2$ or their combination. A study of the expected charge states in the CSC at the FRS-IC is given in [28]. One can measure both charge states in the FRS-IC and combine their contributions. In case of doubt or for newlyinvestigated elements, we will measure the extracted charge states and/or the temporal behavior of stable or near-stable isotopes of the element under investigation.

\section{Results and discussion}

In the following subsections we present results from both off-line ( $\alpha$-recoil source) and on-line (fragment beam) experiments. In all these measurements, the DC pushing field in the CSC was $17 \mathrm{~V} / \mathrm{cm}$ and the RF carpet voltage was $97 \mathrm{~V}_{p-p}$ at a frequency of $5.92 \mathrm{MHz}$.

Off-line measurements were performed with the ${ }^{228} \mathrm{Th}$ $\alpha$-recoil source that is installed inside the CSC (fig. 1). During these measurements, the He gas pressure in the CSC was 33 mbar and its temperature was $74 \mathrm{~K}$, corresponding to an areal density of $2.3 \mathrm{mg} / \mathrm{cm}^{2}$. The decay scheme of this source is shown in fig. 4 .

Online measurements were performed with a $300 \mathrm{MeV} / \mathrm{u}{ }^{238} \mathrm{U}$ primary beam from the heavy-ion synchrotron SIS-18 with an intensity of up to $2.5 \cdot 10^{8}$ ions per spill. A typical spill length of $200 \mathrm{~ms}$ was used, which impinged on a beryllium target with an areal density of $0.270 \mathrm{~g} / \mathrm{cm}^{2}$ to generate fragments. Due to the low primary beam energy the matter in the FRS 


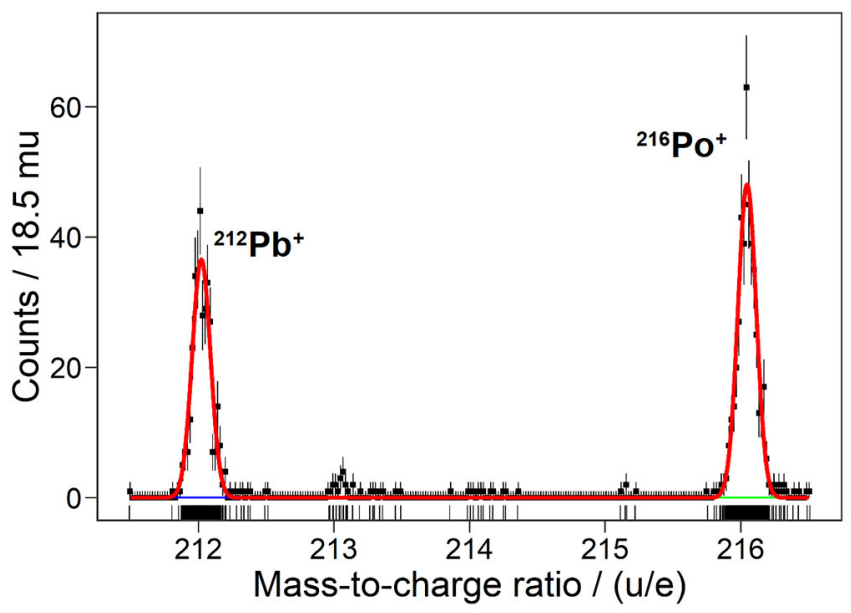

Fig. 5. Mass spectrum from the ${ }^{228}$ Th source installed inside the CSC. The ${ }^{216} \mathrm{Po}$ and ${ }^{212} \mathrm{~Pb}$ isotopes are identified by their mass. At the other mass numbers a small amount of molecular contaminants can be seen, which are formed due to the ionization of the residual gas in the vicinity of the ${ }^{228} \mathrm{Th}$ source. The red line represents the overall fit to the ${ }^{216} \mathrm{Po}$ and ${ }^{212} \mathrm{~Pb}$ isotope peaks. The fit does not take into account the molecular contaminants between them. The un-binned data is shown as well.

beam-line was minimized. The monochromatic degrader at the mid-focal plane of the FRS had an areal density of $737.1 \mathrm{mg} / \mathrm{cm}^{2}$. During these measurements, the $\mathrm{He}$ pressure in the CSC was 64 mbar and the temperature was $77 \mathrm{~K}$, corresponding to an areal density of $4.2 \mathrm{mg} / \mathrm{cm}^{2}$.

A consequence of the low primary beam energy and intensity was that fragment separation was not optimal and signal statistics were generally low, increasing statistical uncertainties.

\subsection{Mass spectra}

In fig. 5 we show a measured mass spectrum of ${ }^{216} \mathrm{Po}$ and ${ }^{212} \mathrm{~Pb}$ isotopes, generated by the ${ }^{228} \mathrm{Th}$ recoil ion source that is installed inside the CSC. The fit curves in fig. 5 are the result of the procedure described in [24]. Similar spectra and their analysis were used at various storage times to measure directly the half-life of the ${ }^{216} \mathrm{Po}$ isotope and to exhibit the simultaneous decay and growth of the mother and daughter nuclides in the $\alpha$-decay of ${ }^{216} \mathrm{Po}$ isotopes (sect. 3.3).

In fig. 6 we show a measured mass spectrum of the ${ }^{119 m 2} \mathrm{Sb}$ isomer, and ${ }^{119 g} \mathrm{Sb},{ }^{119} \mathrm{Te}$ and ${ }^{119} \mathrm{Sn}$ isotopes, resulting from the fragment beam described above. Note that the ${ }^{119} \mathrm{Te}$ and ${ }^{119} \mathrm{Sn}$ isotopes both have long-lived isomeric states $(260.96 \mathrm{keV}, 4.70$ days and $89.531 \mathrm{keV}, 293.1$ days, respectively [29]). The excitation energy of the ${ }^{119} \mathrm{Sn}$ isomeric state is below the FRS-IC resolving power, and although that of the ${ }^{119} \mathrm{Te}$ isotope is in principle resolvable, it was not possible to distinguish it with the low statistics in this work. Therefore, the mass spectra are fitted to a single peak, and the results are compared to the ground state literature values.

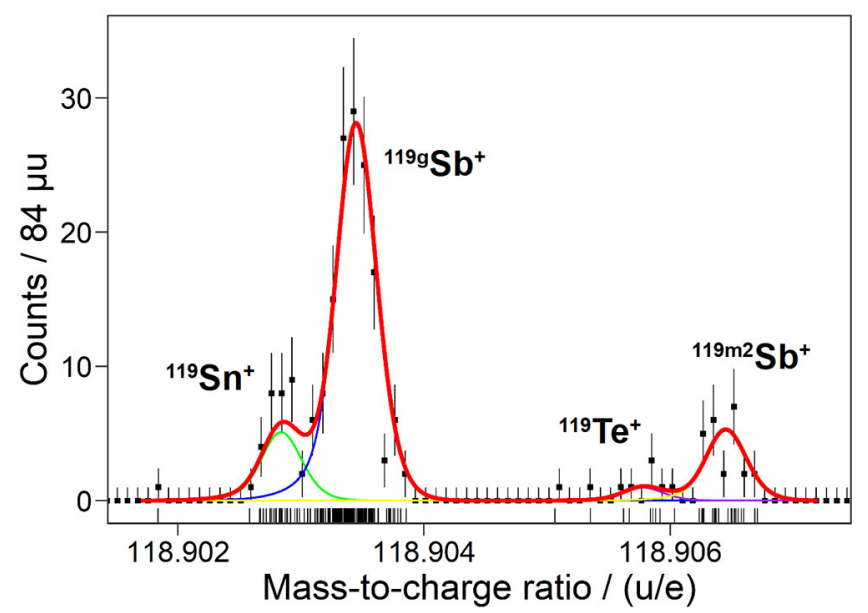

Fig. 6. Mass spectrum of the ${ }^{119 m 2} \mathrm{Sb}$ isomer, and the ${ }^{119 g} \mathrm{Sb}$, ${ }^{119} \mathrm{Te}$ and ${ }^{119} \mathrm{Sn}$ isotopes measured with a mass resolving power of 290,000 . The red solid line represents the overall fit, whereas the green, blue, yellow and pink lines represent fits to the separate isotope peaks, enabling extraction of isotope masses and counts. The ${ }^{119} \mathrm{Te}$ and ${ }^{119} \mathrm{Sn}$ long-living isomeric states are indistinguishable from the respective ground states. The unbinned data is shown as well.

As in fig. 5, the fit curves are from the procedure described in [24]. One can observe the separate peak fits for the distinguishable overlapping peaks $\left({ }^{119 g} \mathrm{Sb}\right.$ and ${ }^{119} \mathrm{Sn}$ isotopes), which give the mass values and the number of counts for each isotope. Use of the Hyper-EMG function [25] proved extremely important for this spectrum, in order to extract the correct amounts for each nuclides, with the lowest uncertainty.

The mass of the ${ }^{119 g} \mathrm{Sb}$ isotope and the excitation energy of the ${ }^{119 m 2} \mathrm{Sb}$ isomer are measured directly for the first time. Our results are given in table 1. The measured masses of the ${ }^{119 g} \mathrm{Sb},{ }^{119} \mathrm{Sn}$ and ${ }^{119} \mathrm{Te}$ isotopes are consistent with the literature values. In sect. 3.2 we compare our ${ }^{119 m}{ }^{2} \mathrm{Sb}$ isomer excitation energy result to the literature and discuss its impact.

\subsection{Insights from the ${ }^{119 \mathrm{~m} 2} \mathrm{Sb}$ isomer mass measurement}

The second isomeric level of ${ }^{119} \mathrm{Sb}$, namely ${ }^{119 \mathrm{~m} 2} \mathrm{Sb}\left(t_{1 / 2}=\right.$ $850 \pm 90 \mathrm{~ms}$ ), was first identified in 1979 [12]. Its excitation energy and spin assignment could not be firmly established so far in spite of multiple attempts [12-15]. This was because of clear methodical problems due to correlations and/or multiple $\gamma$ rays present in the spectra at almost the same energy, as well as the existence of another isomeric state at $2553.6 \pm 0.3 \mathrm{keV}$. The adopted values for ${ }^{119 \mathrm{~m} 2} \mathrm{Sb}$ in the Atlas of Nuclear Isomers [30] and ENSDF [31] are an excitation energy of $2841.7 \pm 0.4+\mathrm{x} \mathrm{keV}$, where $\mathrm{x}$ is to be determined, and spin and parity of $27 / 2^{+}$. The adopted assignment for the $2841.7 \mathrm{keV}$ level is $I^{\pi}=21 / 2^{-}$[31].

Our result for the ${ }^{119 m 2} \mathrm{Sb}$ isomeric state excitation energy, $2799 \pm 30 \mathrm{keV}$ (table 1), is the first experimental data that is based on a direct mass measurement, 
Table 1. Results of direct mass measurements performed at the FRS-IC. The uncertainties shown correspond to the total experimental uncertainty. Literature values are from [29-32]. " $M E$ " stands for Mass Excess, which is reported for ground states. For the ${ }^{119 m 2} \mathrm{Sb}$ isomer we report the excitation energy, $E_{\text {exc }}$. In the MR-TOF-MS, the total time-of-flight was 18.9 ms, and the number of turns was 560 . The reference ion in all these measurements was the ${ }^{12} \mathrm{C}_{2}{ }^{19} \mathrm{~F}_{5}(A=119)$ molecule.

\begin{tabular}{|c|c|c|c|c|c|}
\hline Nuclide & Half-life & $\begin{array}{c}M E_{\mathrm{FRS}-\mathrm{IC}} \\
E_{\text {exc,FRS-IC }} \\
/ \mathrm{keV}\end{array}$ & $\begin{array}{c}M E_{\mathrm{AME} 16} \\
E_{\mathrm{exc}},[30] \\
\quad / \mathrm{keV}\end{array}$ & $\begin{array}{c}M E_{\mathrm{FRS}-\mathrm{IC}}-M E_{\mathrm{AME} 16} \\
E_{\mathrm{exc}, \mathrm{FRS}-\mathrm{IC}}-E_{\text {exc }},[30] \\
/ \mathrm{keV}\end{array}$ & $\begin{array}{l}\text { Number } \\
\text { of events } \\
\text { measured }\end{array}$ \\
\hline${ }^{119 g} \mathrm{Sb}$ & $38.19 \pm 0.22 \mathrm{~h}$ & $-89482 \pm 17$ & $-89474 \pm 8$ & $-8 \pm 19$ & 857 \\
\hline${ }^{119 m 2} \mathrm{Sb}$ & $\begin{array}{c}850 \pm 90 \mathrm{~ms} \\
776 \pm 181 \mathrm{~ms}^{a}\end{array}$ & $2799 \pm 30$ & $2841.7 \pm 0.4+\mathrm{x}$ & $-43 \pm 30$ & 467 \\
\hline${ }^{119} \mathrm{Sn}$ & stable & $-90100 \pm 36$ & $-90065.0 \pm 0.7$ & $-35 \pm 36$ & $237^{b}$ \\
\hline${ }^{119} \mathrm{Te}$ & $16.05 \pm 0.05 \mathrm{~h}$ & $-87310 \pm 177$ & $-87181 \pm 8$ & $-129 \pm 177$ & $25^{b}$ \\
\hline
\end{tabular}

\footnotetext{
${ }^{a}$ Literature and measured value given.

${ }^{b}$ Total number of events for the unresolved ground and isomeric states. The literature values of the ${ }^{119} \mathrm{Sn}$ and ${ }^{119}$ Te isotopes are for their ground states.
}

and is $1.4 \sigma$ below $2841.7 \pm 0.4 \mathrm{keV}$. It suggests that the above-mentioned parameter $\mathrm{x}$ is consistent with 0 , and the $2841.7 \mathrm{keV}$ level itself is the long-lived isomeric state, as was presented originally in ref. [13] (fusion reaction experiment) and adopted in ref. [14] (induced fission experiment).

The experiments of refs. [13, 14] were based on $\gamma$ spectroscopy, and both designated $I^{\pi}=25 / 2^{+}$to the $2841.7 \mathrm{keV}$ level, due to the conversion coefficient value of the $288.2 \mathrm{keV}$ transition depopulating the isomer.

We thus conclude that our result confirms the ${ }^{119 m 2} \mathrm{Sb}$ isomeric state designation of refs. [13,14], in contradiction to the adapted assignment $[30,31]$.

\subsection{Ion storage in the CSC}

We investigated storage in the CSC with the stable and relatively long-living isotopes of the ${ }^{228} \mathrm{Th}$ decay chain from the CSC internal radioactive $\alpha$-recoil source. Our goal was to demonstrate isotope storage in the CSC without (or with negligible) non-nuclear-decay losses (e.g. charge exchange, molecular formation).

We measured the amount of a combination of the ${ }^{208} \mathrm{~Pb}$ isotope (stable) and the ${ }^{208} \mathrm{Tl}$ isotope $\left(t_{1 / 2}=3.053\right.$ minutes) as a function of CSC storage time, which we varied from $0.2 \mathrm{~s}$ up to $9 \mathrm{~s}$. The MR-TOF-MS was set to low mass resolving power, so these two isotopes were not resolved. The red data points in fig. 7 are fitted to a constant (red solid line). ${ }^{220} \mathrm{Rn}\left(t_{1 / 2}=55.6 \mathrm{~s}\right)$ isotopes are also detected in the same measurement. The black data points in fig. 7 , are fitted to an exponential decay curve with the literature half-life (black solid line).

In order to estimate a quantitative limit for nonnuclear losses, we added to the fitted functions a nonnuclear component, from which a non-nuclear decay constant was obtained. From the fit results, we estimate that non-nuclear half-life in the CSC is in the order of a few hundred seconds, having a negligible effect on measure-

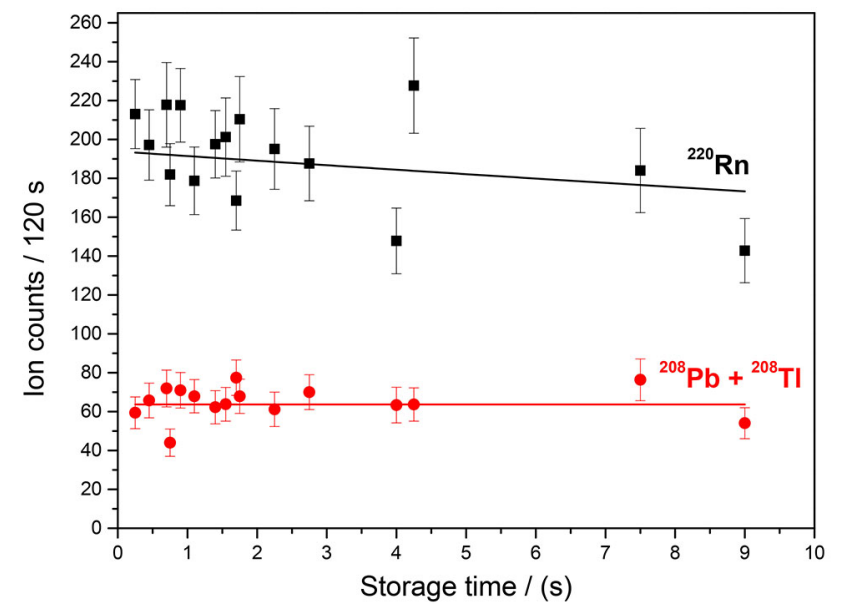

Fig. 7. ${ }^{220} \mathrm{Rn}$ isotope and the sum of ${ }^{208} \mathrm{~Pb}$ and ${ }^{208} \mathrm{Tl}$ isotope counts, as a function of CSC storage time. Solid lines are fits with the literature time constants. The ${ }^{208} \mathrm{~Pb}$ and ${ }^{208} \mathrm{Tl}$ isotope counts are consistent with the expected stable behavior and the ${ }^{220} \mathrm{Rn}$ isotope counts are consistent with the literature value $t_{1 / 2}=55.6 \mathrm{~s}$.

ments of this work, which reached storage times up to about $10 \mathrm{~s}$. This indicates the CSC is extraordinarily clean.

Under the assumption that collisions are dominated by the polarization of the buffer gas [33], the collision rate with the CSC buffer gas (He) is estimated to be $\sim 3 \mathrm{GHz}$ in the experimental conditions of this work. Given our result that the non-nuclear loss time scale is hundreds of seconds (corresponding to a collision rate with impurities of a few $\mathrm{mHz}$ ), the relative impurities level must be lower than $10^{-11}$. This result is consistent with previous measurements in the CSC that demonstrated its cleanliness. For example, see fig. 12 of ref. [18], which shows how impurities in the CSC are reduced as its temperature decreases, whereas the amount of the nuclide of interest stays constant, or even increases (due to increased extraction efficiency). Note that we limited our measurements 
to this storage time due to practical constraints, so the actual upper limit may be higher.

It is important to note here that ionization of the buffer gas of the CSC hardly affects our method. As described in ref. [34], each beam fragment generates $\sim 10^{7}$ He-e pairs. Of these pairs, the fast-moving electrons hit the CSC electrodes and disappear within a few microseconds, and the He ions are removed within a few tens of milliseconds when they hit the RF-carpet. In addition, the RF carpet is set such that all ions with a mass-to-charge ratio less than $\sim 2 / 3$ of the ion of interest are not repelled by the RF field and are also lost. Therefore, for the remainder of the longer storage times that are used in this work, there is no space charge in the CSC and thus no effect on the efficiency.

As is further described in ref. [34], extraction efficiency in the CSC starts to be reduced at an ionization rate of $\sim 10^{11}$ He-e pairs per second, namely rates of $\sim 10^{4}$ beamnuclides/sec. Rates in this work were on the order of $10^{3}$ nuclides/sec, so there was no space-charge effect.

\subsection{Half-life and branching ratio measurement}

We demonstrated simultaneous mother nuclide decay and daughter nuclide growth via the decay of ${ }^{216} \mathrm{Po}$ isotopes $\left(t_{1 / 2}=145 \pm 2 \mathrm{~ms}\right)$ to ${ }^{212} \mathrm{~Pb}$ isotopes $\left(t_{1 / 2}=10.64\right.$ hours $)$, both part of the decay chain of the internal ${ }^{228} \mathrm{Th}$ radioactive recoil source in the CSC.

We plotted the mother and daughter nuclide counts normalized to their sum and performed a global fit on all data points to eqs. (1) and (3), according to the procedure described in sect. 2.4. The relatively long half-life of ${ }^{212} \mathrm{~Pb}$ justified using eq. (3) instead of eq. (2). No a priori assumptions are taken, since we include in the normalization all isotopes whose decay from the investigated mother nuclide have a positive Q-value. For the ${ }^{216} \mathrm{Po}$ isotope, only $\alpha$-decay to the ${ }^{212} \mathrm{~Pb}$ isotope meets this criterion.

In fig. 8 we depict $\alpha$-decay of the ${ }^{216} \mathrm{Po}$ isotope to the ${ }^{212} \mathrm{~Pb}$ isotope in the self-normalizing presentation described above. The ${ }^{212} \mathrm{~Pb}$ isotope counts were corrected for the $50 \%$ loss at the $\mathrm{RF}$ carpet vicinity due to their long range distribution (see sect. 2.2).

It is evident by eye that the two trends are consistent with a mother nuclide decay and its daughter nuclide growth. The results of the global fit in fig. 8 are given in table 2. Per the defined constraint, the sum of $y_{01}$ and $A_{p}$ is consistent with unity. A mother nuclide half-life of $145 \pm 11 \mathrm{~ms}$ is obtained, consistent with the literature value $(145 \pm 2 \mathrm{~ms}[29])$.

To test the effect of the global fit, we also fitted the ${ }^{216}$ Po isotope curve separately to eq. (1), without any constraint with respect to the ${ }^{212} \mathrm{~Pb}$ isotope data. The half-life result in this case is $158 \pm 15 \mathrm{~ms}$, consistent with the global fit results, but with a larger uncertainty. The advantage of including the daughter nuclide data is clear.

In addition, we note that we fitted the un-normalized ${ }^{216} \mathrm{Po}$ isotope data to eq. (1), and also obtained a halflife result that is consistent with the literature value, and

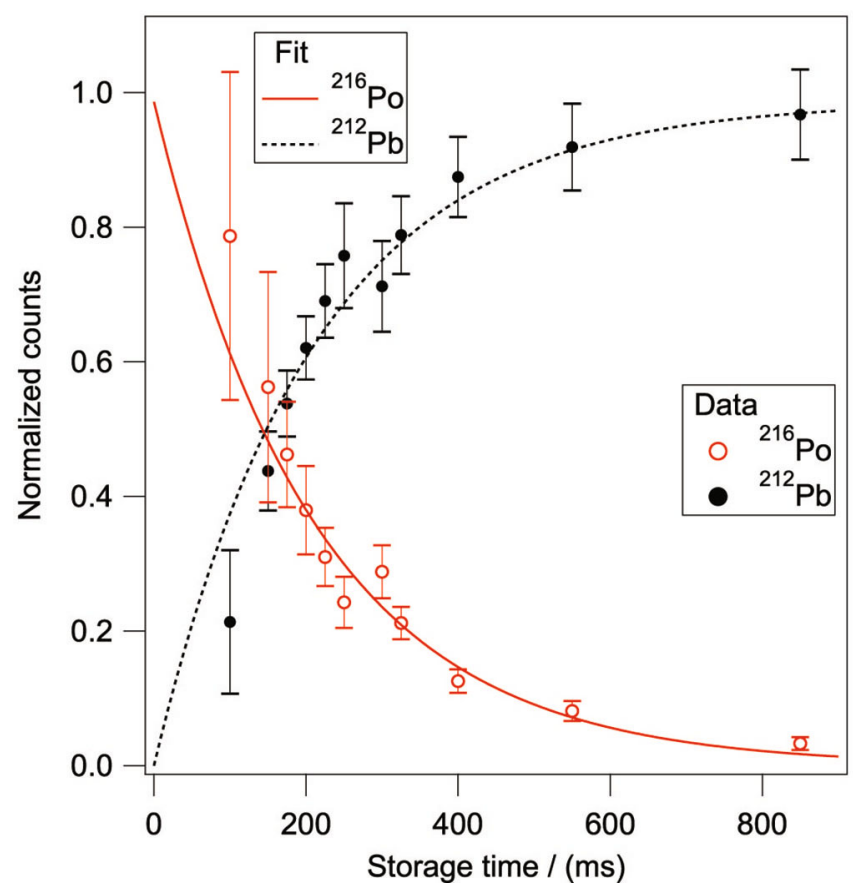

Fig. 8. ${ }^{216} \mathrm{Po}$ and ${ }^{212} \mathrm{~Pb}$ isotope counts, normalized to their sum, as a function of CSC storage time, up to about $1 \mathrm{~s}$. The curves represent a global fit to both data sets to eqs. (1) and (3).

Table 2. ${ }^{216} \mathrm{Po}$ isotope and ${ }^{212} \mathrm{~Pb}$ isotope global fit results for the parameters defined in eqs. (1) and (3).

\begin{tabular}{ccc}
\hline${ }^{216} \mathrm{Po}$ & $A_{P}$ & $1.00 \pm 0.10$ \\
& $t_{1 / 2}[\mathrm{~ms}]$ & $145 \pm 11$ \\
\hline${ }^{212} \mathrm{~Pb}$ & $y_{01}$ & $0.00 \pm 0.10$ \\
\hline
\end{tabular}

with an uncertainty that is similar to the result of the independent fit to the normalized data.

During the measurement described in this work, we experienced a problem with the electronics of the RF carpet that increased the extraction time, which in turn increased the systematic uncertainties at storage times up to $\sim 200 \mathrm{~ms}$. We applied corrections for this problem and dead-time effects in the DAQ, and included the resultant uncertainties in the data analysis.

We further used our method to measure the half-life and decay branching ratios of the ${ }^{119 m} 2 \mathrm{Sb}$ isomer, obtained from the fragment beam. Note, that the problem with the RF carpet electronics did not affect the analysis nor the results described below. For the ${ }^{119 m 2} \mathrm{Sb}$ isomer it is energetically possible to decay to the ${ }^{119} \mathrm{Sn}$ isotope $\left(\beta^{+}\right.$ decay) and the ${ }^{119}$ Te isotope $\left(\beta^{-}\right.$decay $)$, in addition to the $\gamma$-decay to the ${ }^{119 g} \mathrm{Sb}$ isotope. We plotted the normalized counts of the ${ }^{119 m 2} \mathrm{Sb}$ isomer and its energetically possible daughter nuclides. Such a presentation is independent of absolute normalization of the incoming fragment beam from the FRS.

The results are shown in fig. 9. About half of the events given in table 1 were measured in conditions that enable 


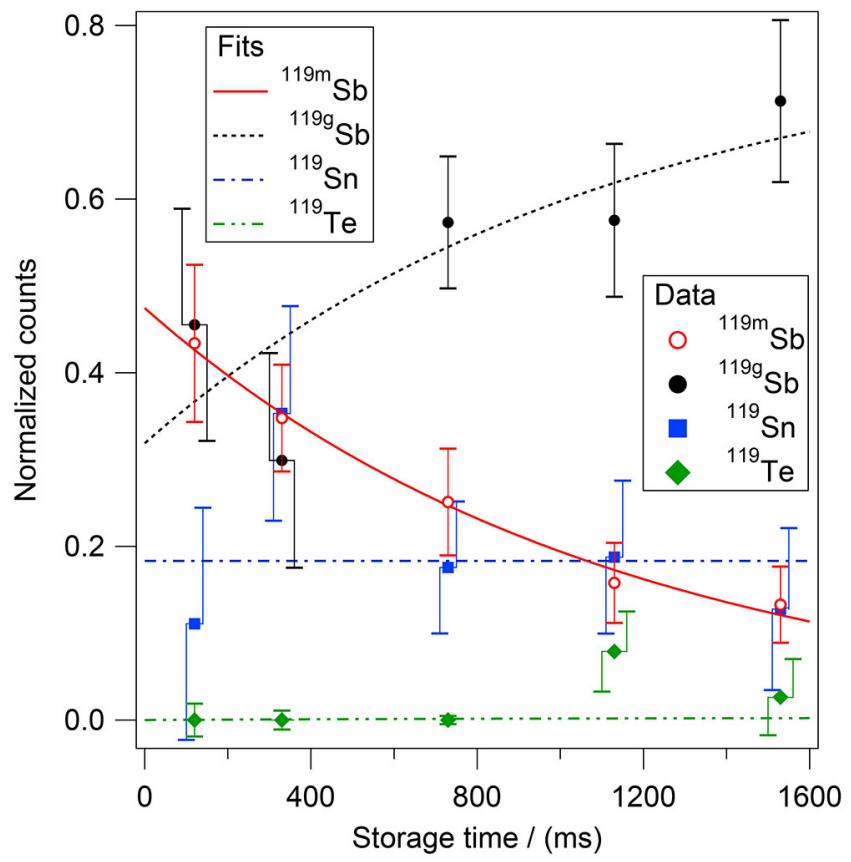

Fig. 9. Normalized counts of the ${ }^{119 m 2} \mathrm{Sb}$ isomer and its energetically possible daughter nuclides, the ${ }^{119 g} \mathrm{Sb}(\gamma$-decay), ${ }^{119} \mathrm{Sn}\left(\beta^{+}\right.$decay $)$and ${ }^{119} \mathrm{Te}\left(\beta^{-}\right.$decay $)$isotopes, as a function of storage time, up to about $1.5 \mathrm{~s}$. Counts of the mother nuclide and the daughter nuclide candidates are normalized to their total sum. The solid lines represent a global fit of all data sets in the figure to eqs. (1) and (3).

Table 3. Global fit results for the ${ }^{119 m 2} \mathrm{Sb}$ isomer decay and all its possible daughter nuclides, for the parameters defined in eqs. (1) and (3).

\begin{tabular}{ccc}
\hline${ }^{119 m 2} \mathrm{Sb}$ & $A_{P}$ & $0.48 \pm 0.08$ \\
& $t_{1 / 2}[\mathrm{~ms}]$ & $784 \pm 203$ \\
\hline${ }^{119 g} \mathrm{Sb}$ & $y_{01}$ & $0.33 \pm 0.12$ \\
& $n_{r 1}$ & $0.99 \pm 0.51$ \\
\hline \multirow{2}{*}{${ }^{119} \mathrm{Sn}$} & $y_{02}$ & $0.19 \pm 0.12$ \\
& $n_{r 2}$ & $0.00 \pm 0.45$ \\
\hline${ }^{119} \mathrm{Te}$ & $y_{03}$ & $0.00 \pm 0.02$ \\
& $n_{r 3}$ & $0.01 \pm 0.08$ \\
\hline
\end{tabular}

contribution to fig. 9. The whole data set is fitted with a global fit to eqs. (1) and (3), according to the procedure described in sect. 2.4. The half-lives of all possible daughter nuclides are long enough to justify not using eq. (2). The fit results are given in table 3.

The ${ }^{119 m 2} \mathrm{Sb}$ isomer half-life given in table 3 is consistent with the literature value $(850 \pm 90 \mathrm{~ms}$ [30]). It is clear that only the ${ }^{119 g} \mathrm{Sb}$ isotope behaves as a daughter nuclide, growing according to eq. (3) with its mother nuclide's half-life (implying branching ratio of unity for $\gamma$ decay), whereas the other two isotopes exhibit a constant temporal behavior, consistent with a branching ratio of zero for $\beta^{+}$and $\beta^{-}$decays.

Our data is thus the first experimental proof that the ${ }^{119 m 2} \mathrm{Sb}$ isomer decays only via $\gamma$-ray emission. Unlike $\gamma$ - spectroscopy experiments that did not observe transitions that are indicative of $\beta^{+}$or $\beta^{-}$decays, we showed positively that there is no growth of the $\beta^{+}$nor $\beta^{-}$daughter nuclides.

We can further extract from table 3 the ${ }^{119} \mathrm{Sb}$ isomer ratio $(I R)$ in the FRS fragment beam, by $I R=\frac{y_{01}}{A_{P}}$. The result we obtain is $0.7 \pm 0.3$.

The global fit with all possible daughter nuclide candidates is not optimal for the extraction of the half-life of the ${ }^{119 m 2} \mathrm{Sb}$ isomer, since it includes input from isotopes that in retrospect have no physical connection to this mother nuclide. It thus merely contributes noise to the fit and increases the uncertainty of the fitted parameters. Therefore, we determine generally that for the extraction of the mother nuclide half-life with the smallest uncertainty, one should repeat the procedure with only the daughter nuclides that have a finite branching ratio. We thus re-fitted only the ${ }^{119 m 2} \mathrm{Sb}$ isomer and ${ }^{119 g} \mathrm{Sb}$ isoope curves, and obtained for the ${ }^{119 \mathrm{~m} 2} \mathrm{Sb}$ isomer a half-life value of $776 \pm 181 \mathrm{~ms}$ (see table 1). This result is consistent with the value in table 3 , and is indeed with a smaller uncertainty.

\section{Further developments and future measurements}

The method presented in this article is well-suited for the measurement of decay branching ratios of nuclei, which decay by more than one channel.

However, as discussed in sect. 2.2, for the method to be operational for all relevant nuclear decay types, we must ensure that the stored isotopes of interest spend the vast majority of their time in the CSC bulk, far away (several $\mathrm{cm}$ ) from any of the CSC electrodes. We describe in this subsection an improvement that will ensure that decays take place mostly within the CSC bulk, thus minimizing daughter nuclide losses on electrodes.

Further in this section, we discuss possible future measurements of half-lives and decay branching ratios with our novel method.

\subsection{Dynamic storage of ions in the CSC bulk}

We have devised a new 3-step scheme for dynamic storage of ions in the CSC bulk, in order to ensure minimal or practically no daughter nuclide loss due to collision with the CSC inner walls. In step 1, the ions from the beam (short pulse, $\sim \mathrm{ms}$ ) are stopped in the CSC, transported via the $\mathrm{DC}$ field to the $\mathrm{RF}$ carpet within twice the mean extraction time $\left(t \sim 2 \times t_{\text {extr }} \sim 50 \mathrm{~ms}\right.$ [16]), and bunched near the extraction nozzle, which is on high potential to block extraction. In step 2, the DC field is switched to a low negative value with respect to the nozzle, and within $10 \mathrm{~ms}$ the isotopes are $1 \mathrm{~cm}$ away from the nozzle. The nuclides diffuse and drift in the weakening electric field towards the CSC center, for a duration controllable from milliseconds to seconds. In step 3, the DC field is switched 


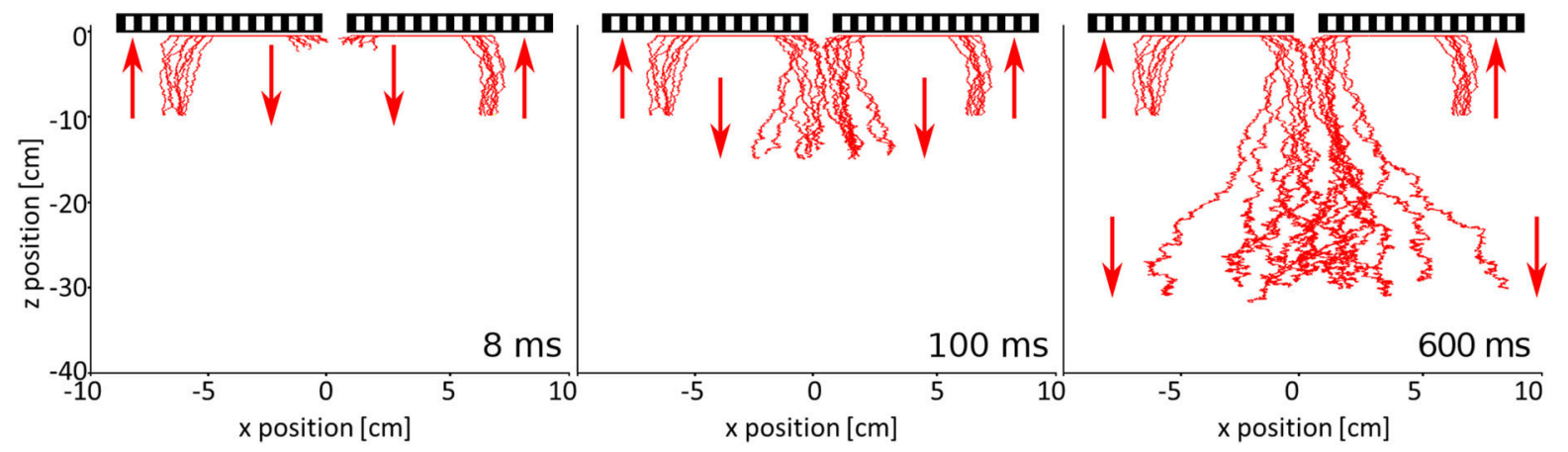

Fig. 10. ITSIM simulation results for dynamic storage in the CSC. Each panel depicts the CSC downstream section. The $\mathrm{RF}$ carpet is marked as a hash. The nozzle diameter is not to scale. The vertical axis $(z)$ is from -40 to $0 \mathrm{~cm}$ (top), and the horizontal axis $(x)$ is from -10 to $+10 \mathrm{~cm}$. The nozzle is at center-top, $z=-0.5 \mathrm{~cm}$. The CSC is $105 \mathrm{~cm}$ long $(z)$ and $25 \mathrm{~cm}$ in diameter $(x)$. Left: from a late part of step 1, to $8 \mathrm{~ms}$ into step 2 , when the isotopes are $\sim 1 \mathrm{~cm}$ away from the nozzle. Middle: time extended to $100 \mathrm{~ms}$ into step 2. Right: ion trajectories from 100 to $600 \mathrm{~ms}$ into step 2 (bulk storage). Incoming and outgoing trajectories are $\mathrm{x}$-separated for clarity. Arrows indicate isotopes general direction.

back to its nominal value and the nozzle potential is lowered, extracting the isotopes from the CSC within a few milliseconds.

Snapshots from a simulation via ITSIM $[35,36]$ of the ion trajectories during the above process are given in fig. 10. The simulation conditions were: He gas at $100 \mu \mathrm{g} / \mathrm{cm}^{3}$, $155 \mathrm{mbar}$, and $80 \mathrm{~K}$, RF carpet voltage of $120 \mathrm{~V}$ peak-to-peak at $6 \mathrm{MHz}$, with a radial DC gradient of $4 \mathrm{~V} / \mathrm{cm}, \mathrm{DC}$ longitudinal field of $10 \mathrm{~V} / \mathrm{cm}$ in step 1 and $-0.5 \mathrm{~V} / \mathrm{cm}$ in step 2 . Ions with mass-to-charge ratio of $219 \mathrm{u}$ /e were used, with a macroscopic collision model, which implies that ion motion through the gas is governed by their mobility value, set to $17.5 \mathrm{~cm}^{2} /(\mathrm{V} \cdot \mathrm{s})$.

The simulation shows that the isotopes are kept at a safe distance from the CSC inner walls for most of their duty cycle. If longer storage times are needed, step 1 and 2 may be cycled. This scheme is possible only because of the small diffusion of the ions in the CSC, which is due to its high pressure and low temperature.

\subsection{Half-lives and decay branching ratio measurements}

The novel measurement method presented here is a powerful mean to investigate masses, half-lives and decay branching ratios of numerous exotic nuclides, in ground and isomeric states, with half-lives ranging from a few tens of milliseconds to a few tens of seconds.

In order to ascertain the range of reachable exotic nuclides in terms of their production rate, we define an "effective production cross section", which is the product of the fragment production cross section, and the branching ratio to the decay channel of interest. Based on the characteristics of the FRS and the FRS Ion Catcher, and seeking a statistical uncertainty of $\sim 15 \%$, we can reach effective production cross sections down the level of $10^{-5}$ mbarn (e.g., for a branching ratio of $\sim 10^{-3}$, the minimal fragment production cross section is $\sim 10^{-2}$ mbarn).

Implementation of new high-resolution and hightransmission modes of the FRS, which have been recently developed [37], may increase the rates of exotic isotopes by a factor of 2, and together with upcoming upgrades of the SIS18 synchrotron, we expect to reach by an order of magnitude larger effective production cross sections. In the future CSC for the Low-Energy Branch of the Super-FRS [38], the reachable effective production cross sections will be extended to $\sim 10^{-8}$ mbarn and in addition, shorter-lived isotopes will be reachable, since the CSC extraction time will be improved to $\sim 5 \mathrm{~ms}$ [39].

Furthermore, branching ratios to spontaneous and $\beta$-delayed fission could be investigated in the future CSC. This is because its density will be as high as $200 \mu \mathrm{g} / \mathrm{cm}^{3}$ [39], enabling almost full fission fragment stopping and storage (the $\sim 100 \mathrm{MeV}$ fission fragments' range distribution will be $\sim 12 \mathrm{~cm}$ ). This will enable unique research opportunities in the field of fission, because our MR-TOF-MS will provide isotope yield distributions and isomer yield ratios, not only mass distributions.

As our method relies on nuclide identification by direct mass measurements, it may be the method of choice for nuclides far away from stability. It can also provide independent and complementary data for exotic nuclides whose properties were measured via gamma de-excitation, but their values (or their physical interpretation) is under debate, e.g. the excitation energy of the isomeric state $119 m 2 \mathrm{Sb}$ discussed in this work.

According to the Atlas of Nuclear Isomers [30], there are at least 20 more isomeric states that could be investigated with our method at the FRS-IC at GSI (ranging from Co to Ra isotopes, with half-lives in the abovementioned region and resolvable excitation energies), whose excitation energy and quantum numbers are still ambiguous. Specific examples include the ${ }^{178} \mathrm{Ta}$ isotope with two such isomeric levels at $1467.82+\mathrm{xkeV}\left(15^{-}\right.$, $58 \mathrm{~ms})$ and $2902.2+\mathrm{x} \mathrm{keV}\left(21^{-}, 290 \mathrm{~ms}\right)$, and the ${ }^{188} \mathrm{Bi}$ and ${ }^{199} \mathrm{Rn}$ isotopes for whom also the branching ratio between $\alpha$ and $\beta^{+}$decays is yet unmeasured.

A particularly interesting isomeric state for investigation with our method is the $6490 \pm 500 \mathrm{keV}$ isomeric state of the ${ }^{94} \mathrm{Ag}$ isotope $\left(21^{+}, 400 \mathrm{~ms}\right)$, which attracted substantial attention as the so-far only nuclear state to decay 
by both one-proton and two-proton direct emission [5], in addition to regular $\beta^{+}$decay, and $\beta$-delayed one- and twoproton emission. This rich variety of decay channels, and the fact that its direct two-proton direct emission channel is still under scrutiny [29,40], makes this nuclide especially attractive for investigation with a new independent method. An experiment that will investigate $N=Z$ nuclei (including the ${ }^{94} \mathrm{Ag}$ isotope) in the FRS Ion Cather is foreseen in the near future $[27,41]$.

$\beta$-delayed nucleon emission may be a field where our method could make a significant impact. For very neutrondeficient nuclides, the direct identification of the daughter nuclides can serve to differentiate between direct and $\beta$ delayed proton emission, especially for cases where the mother nuclide and daughter nuclide candidates are not sufficiently well known to rule out one of the channels due to negative Q-value.

$\beta$-delayed single- and multi-neutron emission is of particular interest to nuclear structure and r-process nucleosynthesis, and is more challenging experimentally than $\beta$-delayed proton emission due to the relative complexity of quantitative neutron detection. There are thus numerous ongoing and near-future campaigns to measure $\beta$-delayed single- and multi-neutron emission branching ratios $[3,10,11,42,43]$, including a planned experiment utilizing our method at GSI [27,44]. This experiment is expected to produce significant results for numerous neutron-rich isotopes, based on recent production cross sections measured at GSI [45].

This new paradigm, of measuring nuclear processes by mass measurements of the outgoing nuclides in an Ion Catcher, may be expanded from decays to reactions. Identifying and counting the outgoing nuclides can be used to infer simultaneously total reaction cross sections of all existing channels for particular projectile-target combinations. A first experiment in this direction is planned for investigating multi-nucleon transfer reactions of ${ }^{238} \mathrm{U}$ ions on several medium- and heavy-ion targets that will be installed in the CSC of the FRS-IC $[27,46]$.

\section{Conclusions}

We present a new method to measure simultaneously masses, isomer excitation energies, Q-values, half-lives and branching ratios, which was developed and demonstrated in the FRS-IC at GSI. The high fragment energy in the FRS makes it the only facility where this method can be implemented. The method includes a first use of a stopping cell as an ion trapping device, for controllable duration from a few tens of ms up to $\sim 10 \mathrm{~s}$.

We demonstrated the method with isotopes from an internal $\alpha$-recoil radioactive source in the stopping cell, and from the FRS fragment beam. For both cases, the expected temporal behavior of mother and daughter nuclides was shown, and half-lives and branching ratios were obtained. The half-lives are consistent with the literature; the branching ratios were measured directly for the first time.
The method requires only very general prior knowledge of the investigated isotopes. This was shown nicely in this work, where while demonstrating the method, we performed the first direct mass measurement of the ground state and second isomeric state of the ${ }^{119} \mathrm{Sb}$ isotope, whose excitation energy and quantum numbers are still not firmly established. Our result for the ${ }^{119 m 2} \mathrm{Sb}$ isomer excitation energy suggests that the isomeric state is the energy level $2841.7 \mathrm{keV}$, with $I^{\pi}=25 / 2^{+}$(based on previous $\gamma$-ray spectroscopy experiments $[13,14])$, in contradiction to the adopted assignment $[30,31]$. We also confirmed positively that $\gamma$ decay is the only branch of the depopulation of this isomeric state.

We have developed a dynamic storage procedure that will ensure isotope storage mainly in the CSC bulk, enabling research of all possible decay channels, including spontaneous and $\beta$-delayed fission.

This method opens the door for a vast range of decay and reaction processes, in an independent and complementary way with respect to existing measurement methods.

This work is part of the doctoral thesis by I. Miskun, Justus Liebig University Gießen, in preparation. This work was supported by the German Federal Ministry for Education and Research (BMBF) under contracts no. 05P12RGFN8 and 05P15RGFN1, by Justus-Liebig-Universität Gießen and GSI under the JLU-GSI strategic Helmholtzpartnership agreement, by HGS-HIRe, and by the Hessian Ministry for Science and Art (HMWK) through the LOEWE Center HICforFAIR. I. Mardor acknowledges support from the Israel Ministry of Energy, Research Grant No. 217-11-023.

Data Availability Statement This manuscript has no associated data or the data will not be deposited. [Authors' comment: All data generated during this study are contained in this published article.]

Publisher's Note The EPJ Publishers remain neutral with regard to jurisdictional claims in published maps and institutional affiliations.

Open Access This is an open access article distributed under the terms of the Creative Commons Attribution License (http://creativecommons.org/licenses/by/4.0), which permits unrestricted use, distribution, and reproduction in any medium, provided the original work is properly cited.

\section{References}

1. C. Iliadis, Nuclear Physics of Stars (Wiley-VCH Verlag $\mathrm{GmbH} \& \mathrm{Co}, 2015)$.

2. M.J.G. Borge, Phys. Scr. 2013, 014013 (2013).

3. I. Dillmann, A. Tarifeo-Saldivia, Nucl. Phys. News 28, 28 (2018).

4. G. Lorusso, A. Becerril, A. Amthor, T. Baumann, D. Bazin, J.S. Berryman, B.A. Brown, R.H. Cyburt, H.L. Crawford, A. Estrade et al., Phys. Rev. C 86, 014313 (2012). 
5. I. Mukha, E. Roeckl, L. Batist, A. Blazhev, J. Döring, H. Grawe, L. Grigorenko, M. Huyse, Z. Janas, R. Kirchner et al., Nature 439, 298 (2006).

6. T. Eronen, A. Kankainen, J. Äystö, Prog. Part. Nucl. Phys. 91, 259 (2016)

7. A. Herlert, S. Van Gorp, D. Beck, K. Blaum, M. Breitenfeldt, R.B. Cakirli, S. George, U. Hager, F. Herfurth, A. Kellerbauer et al., Eur. Phys. J. A 48, 97 (2012).

8. R. Wolf, D. Atanasov, K. Blaum, S. Kreim, D. Lunney, V. Manea, M. Rosenbusch, L. Schweikhard, A. Welker, F. Wienholtz et al., Nucl. Instrum. Methods Phys. Res. B 376, 275 (2016).

9. B. Franzke, H. Geissel, G. Münzenberg, Mass Spectrom. Rev. 27, 428 (2008).

10. H. Miyatake, M. Wada, X.Y. Watanabe, Y. Hirayama, P. Schury, M. Ahmed, H. Ishiyama, S.C. Jeong, Y. Kakiguchi, S. Kimura et al., AIP Conf. Proc. 1947, 020018 (2018).

11. R.M. Yee, N.D. Scielzo, P.F. Bertone, F. Buchinger, S. Caldwell, J.A. Clark, C.M. Deibel, J. Fallis, J.P. Greene, S. Gulick et al., Phys. Rev. Lett. 110, 092501 (2013).

12. R.E. Shroy, A.K. Gaigalas, G. Schatz, D.B. Fossan, Phys. Rev. C 19, 1324 (1979).

13. S. Lunardi, P.J. Daly, F. Soramel, C. Signorini, B. Fornal, G. Fortuna, A.M. Stefanini, R. Broda, W. Meczynski, J. Blomqvist, Z. Phys. A 328, 487 (1987).

14. M.G. Porquet, T. Venkova, R. Lucas, A. Astier, A. Bauchet, I. Deloncle, A. Prévost, F. Azaiez, G. Barreau, A. Bogachev et al., Eur. Phys. J. A 24, 39 (2005).

15. C.B. Moon, G.D. Dracoulis, R.A. Bark, A.P. Byrne, P.A. Davidson, T. Kibedi, G.J. Lane, A.N. Wilson, J. Korean Phys. Soc. 59, 1539 (2011).

16. W.R. Plaß, T. Dickel, S. Purushothaman, P. Dendooven, H. Geissel, J. Ebert, E. Haettner, C. Jesch, M. Ranjan, M.P. Reiter et al., Nucl. Instrum. Methods B 317, 457 (2013).

17. H. Geissel, P. Armbruster, K. Behr, A. Brünle, K. Burkard, M. Chen, H. Folger, B. Franczak, H. Keller, O. Klepper et al., Nucl. Instrum. Methods B 70, 286 (1992).

18. M. Ranjan, P. Dendooven, S. Purushothaman, T. Dickel, M. Reiter, S. Ayet San Andrés, E. Haettner, I. Moore, N. Kalantar-Nayestanaki, H. Geissel et al., Nucl. Instrum. Methods A 770, 87 (2015).

19. T. Dickel, W.R. Plaß, A. Becker, U. Czok, H. Geissel, E. Haettner, C. Jesch, W. Kinsel, M. Petrick, C. Scheidenberger et al., Nucl. Instrum. Methods A 777, 172 (2015).

20. T. Kubo, Nucl. Instrum. Methods Phys. Res. B 204, 97 (2003)

21. O.B. Tarasov, D. Bazin, Nucl. Instrum. Methods Phys. Res. B 266, 4657 (2008).

22. N. Iwasa, H. Geissel, G. Münzenberg, C. Scheidenberger, T. Schwab, H. Wollnik, Nucl. Instrum. Methods Phys. Res. B 126, 284 (1997).

23. J.F. Ziegler, M. Ziegler, J. Biersack, Nucl. Instrum. Methods Phys. Res. B 268, 1818 (2010).

24. S. Ayet San Andrés, C. Hornung, J. Ebert, W.R. Plaß, T. Dickel, H. Geissel, C. Scheidenberger, J. Bergmann, F. Greiner, E. Haettner et al., Phys. Rev. C 99, 064313 (2019).

25. S. Purushothaman, S. Ayet San Andrés, J. Bergmann, T. Dickel, J. Ebert, H. Geissel, C. Hornung, W.R. Plaß, C. Rappold, C. Scheidenberger et al., Int. J. Mass Spectrom. 421, 245 (2017).

26. H. Bateman, Proc. Cambridge Philos. Soc. 15, 423 (1910).
27. W.R. Plaß, T. Dickel, I. Mardor, S. Pietri, H. Geissel, C. Scheidenberger, D. Amanbayev, S. Ayet San Andrés, J. Aysto, D. Balabanski et al., to be published in Hyperfine Interact. (2019).

28. I. Miskun, A.K. Rink, F. Greiner, S. Ayet San Andrés, S. Bagchi, J. Bergmann, P. Constantin, T. Dickel, J. Ebert, A. Finley et al., GSI Sci. Rep. 2017-1, 174 (2017).

29. G. Audi, F.G. Kondev, M. Wang, W.J. Huang, S. Naimi, Chin. Phys. C 41, 030001 (2017).

30. A.K. Jain, B. Maheshwari, S. Garg, M. Patial, B. Singh, Nucl. Data Sheets 128, 1 (2015).

31. D. Symochko, E. Browne, J. Tuli, Nucl. Data Sheets 110 2945 (2009) data extracted from the ENSDF database, version 2019-02-14.

32. M. Wang, G. Audi, F.G. Kondev, W.J. Huang, S. Naimi, X. Xu, Chin. Phys. C 41, 030003 (2017).

33. H.G. Dehmelt, Advances in Atomic and Molecular Physics, Vol. 3, edited by D.R. Bates, I. Estermann (Academic Press, 1968) pp. 53-72.

34. M.P. Reiter, A.K. Rink, T. Dickel, E. Haettner, F. Heiße, W.R. Plaß, S. Purushothaman, F. Amjad, S. Ayet San Andrés, J. Bergmann et al., Nucl. Instrum. Methods B 376, 240 (2016).

35. G. Wu, R.G. Cooks, Z. Ouyang, M. Yu, W.J. Chappell, W.R. Plaß, J. Am. Soc. Mass Spectrom. 17, 1216 (2006).

36. W. Plaß, T. Dickel, B. Fabian, E. Haettner, An advanced concept for mass spectrometry simulations - The ion trajectory simulation program ITSIM 6, in Proceedings of the 56th ASMS Conference on Mass Spectrometry and Allied Topics, Denver, Colorado, USA (ASMS, 2008) MP 015.

37. E. Haettner, B. Franczak, H. Geissel, T. Dickel, S. Pietri, W. Plaß, A. Prochazka, S. Purushothaman, C. Scheidenberger, Y. Tanaka et al., Nucl. Instrum. Methods Phys. Res. B, https://doi.org/10.1016/j.nimb.2019.04.025.

38. H. Geissel, H. Weick, M. Winkler, G. Münzenberg, V. Chichkine, M. Yavor, T. Aumann, K.H. Behr, M. Böhmer, A. Brünle et al., Nucl. Instrum. Methods Phys. Res. B 204, 71 (2003).

39. T. Dickel, W.R. Plaß, H. Geissel, F. Heiße, I. Miskun, S. Purushothman, M.P. Reiter, A.K. Rink, C. Scheidenberger, Nucl. Instrum. Methods Phys. Res. B 376, 216 (2016).

40. A. Kankainen, V.V. Elomaa, L. Batist, S. Eliseev, T. Eronen, U. Hager, J. Hakala, A. Jokinen, I.D. Moore, Y.N. Novikov et al., Phys. Rev. Lett. 101, 142503 (2008).

41. W.R. Plaß, T. Dickel, S. Ayet San Andrés, S. Bagchi, D. Balabanski, S. Beck, P. Constantin, T. Eronen, H. Geissel, F. Greiner et al., GSI Sci. Rep. 2018-1, 115 (2018).

42. A. Evdokimov, I. Dillmann, M. Marta, F. Bosch, A. Dolinskii, C. Kozhuharov, Y. Litvinov, F. Nolden, M. Steck, H. Weick et al., PoS NIC XII, 115 (2012).

43. IAEA CRP on a Reference Database for BetaDelayed Neutron Emission, https://www-nds.iaea.org/ beta-delayed-neutron (2017).

44. I. Mardor, T. Dickel, S. Ayet San Andrés, S. Bagchi, S. Beck, H. Geissel, F. Greiner, E. Haettner, C. Hornung, D. Kostyleva et al., GSI Sci. Rep. 2018-1, 114 (2018).

45. D. Pérez-Loureiro, J. Benlliure, J. Díaz-Cortes, J.L. Rodríguez-Sánchez, H. Álvarez-Pol, B. Blank, E. Casarejos, D. Dragosavac, V. Föhr, M. Gascón et al., Phys. Rev. C 99, 054606 (2019).

46. T. Dickel, P. Constantin, J. Winfield, S. Ayet San Andrés, S. Bagchi, D. Balabanski, S. Beck, H. Geissel, F. Greiner, E. Haettner et al., GSI Sci. Rep. 2018-1, 112 (2018). 